\title{
Planning Simulation Experiments in the Tasks of Studying the Operational Strategies of Container Terminals
}

\author{
A.L. Kuznetsov, A.V. Kirichenko \& A.D. Semyonov \\ Admiral Makarov State University of Maritime and Inland Shipping, Saint-Petersburg, Russia \\ H. Oja \\ Konecranes Finland Corp., Hyvinkä̈̈, Finland
}

\begin{abstract}
There is a lot of scientific papers that consider the efficiency of different container yard's handling strategies. The methods of assessment vary from abstract mathematical calculations to aposterioric collection and analyses of practical data. This article deals with a new way of stacking strategy's estimation. It postulates that investigations based on the extrapolation of existing strategies cannot be reliable due to rapid changes in terminals' environment and restricted amount of data that can be collected within limited periods of the strategies' application. The hypothesis of the study is that the only method that could provide robust comparative study of container stacking strategies is the simulation modelling. In the same time, any model is only a tool of analyses. The synthesis could be provided only by massive iterations of single simulation experiments with controlled searching procedure in the space of parameters. Consequently, the stress of the simulation study should be put not on the model itself, but on the way how to use it, i.e. on the experiments planning. Only a rationally constructed machine of simulation scenario generation could provide adequate and statistically reliable results. In order to demonstrate the importance of this 'task setting' machine, two example strategies are selected for comparison: (1) allocation of containers to slots with the minimum height and (2) stacking of containers in accordance with expected dwell time. It also shows that the objective function has a great impact on the optimal strategy implementation. In the study described in the paper the number of moves requested to select a container is opted as an optimization criterion.
\end{abstract}

\section{INTRODUCTION}

The laboriousness (complexity) of container handling operations is usually evaluated by the average number of moves required for their performing. In order to calculate the commercial and operational characteristics one also needs to consider the time needed to implement each movement [1-4]. When the analytical approach is used, one cannot think in terms of single operations, so the mean time of moves is usually considered instead. Still, the number of moves is not deterministic but stochastic value and should be described by distribution functions. In order to get ones, e.g. distribution density, which is important parameter for container terminal design and operational management, usually methods of MonteCarlo group are used [5]. These methods, actually, can be considered as an attempt to introduce individual values of different parameters which are defined by the integral functions of each characteristic. In a way, these methods of random generation of values are directly reversed to the generalization provided the step before.

In many cases this approach can be considered as oversimplified, i.e. when a terminal exploits several different types of container handling equipment for stacking operations. Another problem appears when 
one wishes to study technological and operational changes which can manifest themselves and affect the terminals' efficiency only in a long-termed perspective.

Generally speaking, the operational strategy of the cargoflow handling consists of few particular tactics. From mathematical point of view, most of these tactics should be treated as heuristics. The "heuristics" is used in operational research theory to describe methods which are considered to be useful and effective, but these features cannot be proved mathematically [6-7]. As the result, their right for existing is only proved by practice and/or simulation modelling.

The heuristics constituting the operational strategy are usually relevant to different interconnected parts of general problem, that is why their individual influence on the quality of operational decisions very is difficult to separate and assess. The complex interaction of different elements and unknown sensitivity of the results to stochastic fluctuations of the parameters cause high volatility of the final evaluation. A single experiment cannot neither prove any conclusions nor reveal the effects of such "weak" influences. One example of these influences is the 'operational temperature' of a box in the stack. This particular factor is selected in the paper to unfold the suggested method of simulation experiments' planning.

\section{THE GOAL SETTING OA PARTIAL STRATEGY STUDY}

As it was mentioned above, the operational strategies of container terminal are usually formed from the number of heuristics and thus based on intuitive perceptions. One of the most famous examples of this perception is commonly practiced segregation of containers on the "hot" and "cold" ones. This segregation is based on the consideration of containers dwell time.

In the practice of container yard operations, the "hot" containers are the ones that dwelled in the stack long enough to be selected from the stack soon. Otherwise, recently arrived containers are the "cold" ones and expected to dwell there for some time. "Cold" containers arrive after "hot" and could block the access to the "hot" ones, so they have to be shifted to other positions in order to clear a target "hot" container. An example of a stack where "cold" and "hot" containers marked by different colors reflecting their current dwell time is represented by Fig. 1.

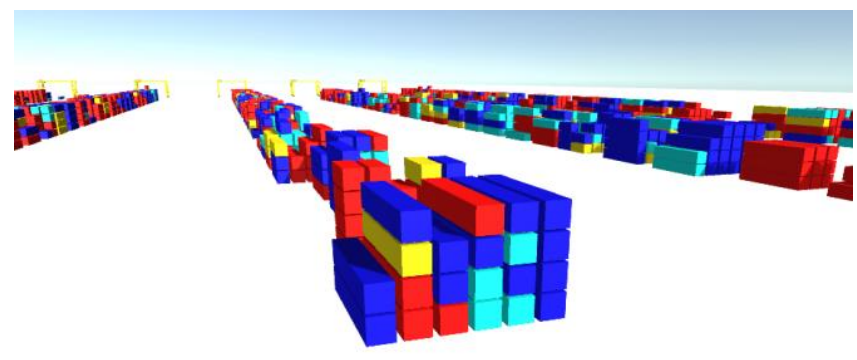

Figure 1. An example of graphical representation of containers' status

When a request for container selection occurs, the target container can be blocked by other ones. Traditionally, the blocking container is replaced to closest position with minimum height [8-9]. From the other hand, the higher the temperature of blocking container, the higher is the probability that it will soon leave the terminal. Consequently, "hot" containers could be moved to higher positions, where they would not be blocked by other containers. At the same time, it can take a longer time to place a container to a position chosen by "operational temperature" then to closest one with minimal height, since it could require longer travel distance. This could lead to the decrease of the positive effect gained from the number of moves reduction due to increase of mean move time. The results can be proved or denied only by very large numbers of experiments. However, envisaging the subtle character of this effect, these experiments must be implemented simultaneously for each variant of container stacking strategy in absolutely identical operational conditions.

Implementing this or that technological innovations in the operational practice of a real terminal, we will not be able to make any evidential conclusions: the registered changes of parameters could be influenced by many controllable and uncontrollable factors, including pure statistical errors caused by limited data base of real world experiments. If, for example, we would read over every arriving container surah of Quran or sprinkle with Holy Water or apply semantical networks for improving the allocation, to evaluate the effect of these techniques by the operational data in the end of the year will be impossible. In order to do so we had to gain the parallel results from these three hypothetic variants and the forth basic variant, where we would operate without proposed measures, and do it in absolutely identical external operational environment (mainly on the same patterns of external transport flows). Moreover, the closer would be the monitoring results, the longer period of observation it will take. Obviously, the practical experiments with real terminals are inacceptable, both for 'singularity' of the results and thus low statistical reliability, and simply for costs of these experiments. On the other hand, equally inapplicable turn out to be also common simulation techniques.

The methodological problem responsible for this contradiction is that every task of the study of a partial strategy requires the development and implementation of specific simulation models [10]. 
These partial models should be synchronized and coordinated by input data, together creating a unified basis for adequate and efficient study of various operational strategies. This paper offers a variant to solve this problem.

\section{THE MODEL OF SCENARIO GENERATION}

The import containers, arriving by sea and departing by land, move opposite to export containers, arriving by land and departing by sea. In both directions this movement is not continuous and ceaseless; it assumes delays of containers on the terminal (dwelling or storage) for certain time (Fig. 2).

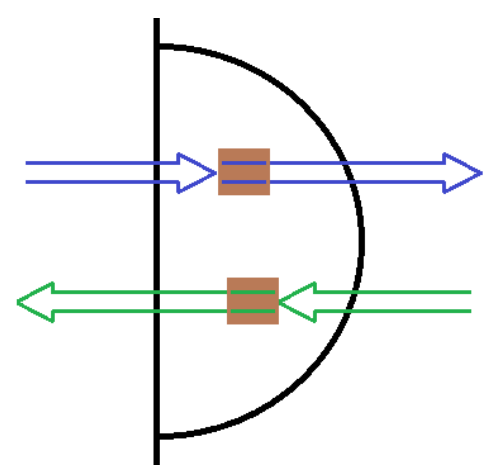

Figure 3. Graphical representation of container flows and storage

Every crossing of the terminal perimeter by cargo flows shown by this figure assumes certain physical handling of containers, mainly discharging from arriving and loading on the departing vehicles. The relevant facilities constitute the dedicated cargo front. Depending on the capacity of the vehicles handled at this front, the discharging and loading procedures deal with different sizes of compact cargo parties. For the automobile transport they encounter couples of containers, for rail transport - hundreds and for sea transport - several thousands

Obviously, all containers arrived to the terminal sooner or later leave it, so the algebraic sum of all inbound flows taken with the sign plus and all outbound ones with the sign minus is zero. In the same time, the typical cargo parties of different transport modes cause different "packs" of containers in inbound and outbound flows. For example, an ocean ship delivered several thousand containers to a port should be discharged and charged as soon as possible to minimize its idle operation time, i.e. cargo operation time for port operators and ship turnaround time for ship owners.

A cargo party of a ship could consist of hundreds railroad train parties, receiving and dispatching of which should be done so that the railroad routes' capacity is not exceeded. Similarly, the truck traffic should be spread evenly in order to meet the throughput capacity of the connecting road network.

The unevenness of the inbound and outbound flows caused by the different sizes of cargo parties and requirements to handle them in different periods of time necessitates the existence of certain 'buffer stock' of containers at the terminal. The physical storage of these containers is performed by the container yard of the terminal. The container yard is characterized by its capacity, handling technology and equipment parameters, which are defined by the characteristics of container flows.

These dependences reveal themselves both directly and indirectly, through many internal ties (e.g. by the limited size of the technologic resources shared for different operations). Their specific interdependences make analytical (algebraic) techniques inadequate to provide the accuracy needed for the design and planning activity, thus forcing to use mathematical modeling, particularly simulation.

The general structure of the model complex (environment) designated to serve as a tool of the sea port operation analyses is given by Fig. 3 .

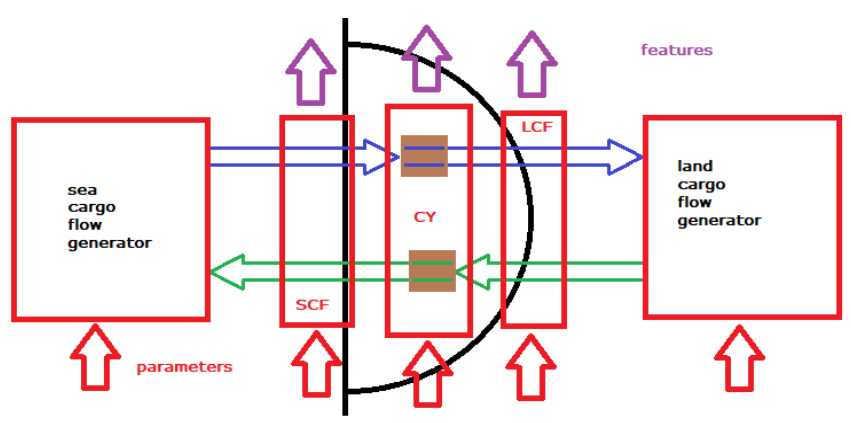

Figure 3. General structure of the sea terminal model environment

The model shown by this figure consists of two generators of cargo flows (sea side and land side), the sea cargo front (SCF), the land cargo front (LCF) and the container yard (CY).

The parameters of container flows include annual volumes, the sizes of cargo parties and time characteristics (unevenness and densities). The parameters of cargo fronts describe their throughput capacities expressed via the productivity and number of technological equipment allocated for handling. The parameters of the container yard include one-time storage capacity and productivities of receptiondispatching operations.

The features of the model, specifically the characteristics of elements' performance, should be studied not qualitatively, but quantitatively, as well as the time characteristics of the technologic processes running inside it (utilization coefficients, delays and refuses of servicing, queue lengths, waiting times etc.). It is important to note that all input and output parameters are not determined, but random values and are represented by their a-priori and a-posteri distribution functions.

Well known is the fact that the simulation is an instrument of analyses and not of syntheses. Any simulation enables to assess the qualitative characteristics of the simulated object depending on its input parameters' values. Since all these parameters are random values, it is necessary to run serial experiments in numbers which would enable to guarantee the required degree of the statistical reliability.

Besides the quantitative parameters set by deterministic and random values, the handling of 
cargo flows passing through the terminal depends on the different strategies of containers' allocation on the yard aiming on the reduction of selection time, minimization of idle moves, and maximization of the utilization of the technological resources. The selection of the appropriate strategy also could be done using dedicated logical reference machines. This feature allows to use this simulation tool not only for accessing the values of the technological parameters, but for comparative study of operational strategies and tactics.

When using the simulation model not as the design tool, but as an instrument for operational planning, the sea side and land side generators would be replaced by real-time information of vehicles arriving and leaving within the planning interval of time, as well as the actual data on the state of the technological processes running at the terminal's elements, i.e. cargo fronts and yards. In this case the models serve as a tool for the sensitivity analyses ('what happens if ...') in order to seek for rational distribution of the technological resources between different operations.

\section{RESULTS AND DISCUSSIONS}

The single run of the model external generator shown in Fig. 3 provides the detailed and full annual time schedules for every transport mode connected in the node. These time schedules contain the exact arrival and departure times for every container passing through the terminal within the simulation term. Arriving containers should be allocated in the stack of container yard, and in due moment (determined by the generated time schedules) would leave the terminal. As it was said above, in this paper the investigated function of the operational strategy is the shifting of blocking containers not in the lowest positions (slots), but in spots determined taking into account the operational temperature (the rest of dwell time). The criterion for the estimation is the laboriousness of selection. For this purpose, during the simulation the total number and number of moves per box are calculated. The computation is being done by simulation of every physical motions and moves of technologic equipment operating in the container yard area.

The functional simulation model of container yard operation in the structure of the modelling environment on Fig. 3 is represented by the rectangular in the center marked as ' $\mathrm{CY}^{\prime}$. The arrow denoting the model parameters includes both geometrical characteristics of the container yard area and technological features of the container handling system, qualitative and quantitative. The discriminating parameter of the investigated function of the strategy are also included into this set: in one case it is the minimal height strategy, in the other minimal operational temperature.

The difference in the number of movements received as the investigated property on Fig. 3, enables to make judgment on the advantage or disadvantage of the strategy over random allocation.
In order to make the statistically reliable conclusions every experiments were repeated many times for different variants of container flows structures and volumes. This enables to exclude any statistical fluctuations and influence of different patterns of external transport arrival.

Fig. 4 represents the results of the simulation set undertaken for the study of the selected function of operational strategy.

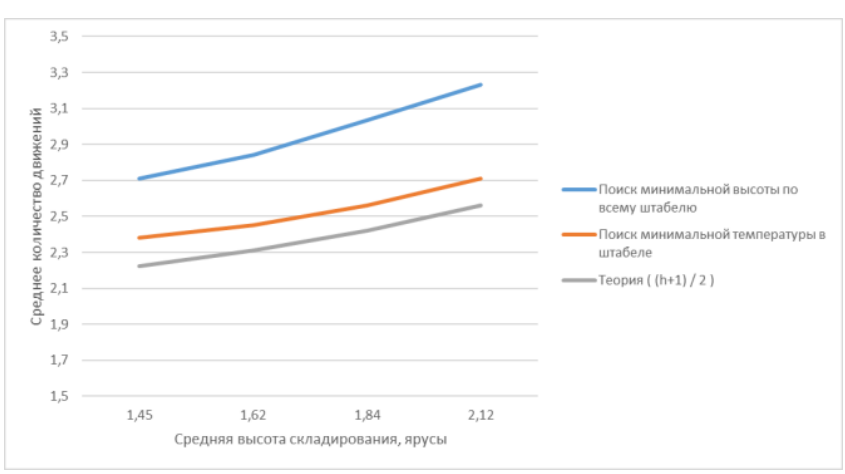

Figure 4. Results of experiment

The basic theoretical complexity of selection is computated by the simple combinatoric formula $\frac{n+1}{n}$ the lines showing the complexity of minimal height and minimal temperature strategies show the saving of 0.5 moves per container in favor of the latter. Though this gain turns out to be big enough in the year term, to reveal this fact by any traditional singular simulation would not be possible.

\section{CONCLUSIONS}

1 Basic components of container terminal operational strategies are the heuristics, or the methods whose efficiency cannot be proved theoretically.

2 In order to include a heuristic into operational strategy of container terminal, it is necessary to prove its practical usefulness, which cannot be done only by imitational simulation.

3 The construction of the scenarios of influencing conditions needed to investigate a strategy requires to take into account many real factors, like volumes and structures of cargo flows, sizes of cargo parties, irregularity of the traffic, throughput capacity of elements etc.

4 In order to receive the statistically reliable results, the experiments should be done in large numbers, both for stochastically close versions and different variants, all in the same environment and with identical values.

5 The paper offers the methodic for controlled generation of scenarios, turning the simulation tool from the instrument of analyses into the instrument of synthesis.

6 As an example, showing the efficiency of the proposed technique the strategic function of container allocation by the current rest of the dwell time is selected. 


\section{REFERENCES}

[1] Kuznetsov A.L., Kirichenko A.V., Semenov A.D.: Box Selectivity in Different Container Cargo-handling Systems. TransNav, the International Journal on Marine Navigation and Safety of Sea Transportation, Vol. 13, No. 4, doi:10.12716/1001.13.04.12, pp. 797-801, 2019

[2] Kuznetsov A.L., Kirichenko A.V., Semenov A.D.: Evaluation of Sinking Effect in Container Stack TransNav, the International Journal on Marine Navigation and Safety of Sea Transportation, Vol. 14, No. 1, doi:10.12716/1001.14.01.19, pp. 159-162, 2020

[3] Kuznetsov K., Kirichenko A., Semenov A., Borevich A., Optimization Strategies of Container Terminals, Scientific Journal of Gdynia Maritime University, No. 115 , pp. 42-54, 2020

[4] Kuznetsov A., Kirichenko A., Izotov O. The Influence of the Storage Strategy on the Complexity of the Container Selection Procedure //IOP Conference Series: Earth and Environmental Science. - 2018. - Vol. 171. - No. 1. - pp. $1755-1315$
[5] Hammersley J. Monte Carlo methods. - Springer Science \& Business Media, 2013.

[6] Sanen Y. A., Dekker R. Intelligent stacking as way out of congested yards part 2 //Port Technol Int 2007. - 2007. Vol. 32. - pp. $80-85$

[7] Borgman B. Online rules for container stacking / B. Borgman, E. van Asperen, R. Dekker //OR spectrum. 2010. - Vol. 32. - Is. 3. - pp. 687-716.

[8] Dekker R., Voogd P., Van Asperen E. Advanced methods for container stacking //Container terminals and cargo systems. - Springer, Berlin, Heidelberg, 2007. - pp. 131154.

[9] Böse, Jürgen W., ed. Handbook of terminal planning. Vol. 49. Springer Science \& Business Media, 2011

[10] Dragović B. Simulation modelling in ports and container terminals: literature overview and analysis by research field, application area and tool / B. Dragović, E. Tzannatos, N. K. Park // Flexible Services and Manufacturing Journal. - 2017. - Vol. 29. - Is. 1. pp. 4-34. DOI: 10.1007/s10696-016-9239-5. 